Jpn J Human Genet 42, 417-423, 1997

\title{
A COMPREHENSIVE METHOD TO SCAN FOR POINT MUTATIONS OF THE GLUCOSE 6 PHOSPHATE DEHYDROGENASE GENE
}

\author{
Taku Shirakawa, ${ }^{1}$ Kaoru Nishryama, ${ }^{1}$ Lai PoH-San, ${ }^{2}$ \\ and Masafumi MATSUO ${ }^{3, *}$ \\ ' Faculty of Health Science, Kobe University School of Medicine, \\ Tomogaoka, Suma-ku, Kobe 654-01, Japan \\ ${ }^{2}$ Department of Pediatrics, National University of Singapore, \\ Lower Kent Ridge Road, Singapore \\ ${ }^{3}$ Division of Genetics, International Center for Medical Research, \\ Kobe University School of Medicine, Kusunoki-cho, Chuo-ku, Kobe 650, Japan
}

\begin{abstract}
Summary We have developed a fast and comprehensive method to scan for point mutations in a gene on $\mathrm{X}$ chromosome. A target region of the gene is first amplified. Then, using the amplified product as a template, PCR is carried out with multiple short-length forward primers arrayed in tandem in the scanned region, and a common reverse primer. The absence of amplified product defines the site of a mutation within a narrow region of the primer recognition site. To evaluate our method, point mutations in exon 12 of the human glucose-6-phosphate dehydrogenase (G6PD) gene were used as a model system. Out of 12 Singaporean G6PD-deficient patients, 6 cases were shown by the method to have a nucleotide change in this exon. Sequence analysis confirmed the presence of a nucleotide change in the region identified by our scanning. Thus, our method is accurate in localizing mutations within a narrow region, and allows large numbers of samples to be handled simultaneously.
\end{abstract}

Key Words molecular diagnosis, multiplex PCR, G6PD deficiency

\section{INTRODUCTION}

A growing number of genes responsible for human diseases have been cloned and analyzed for the presence of mutations. The majority of the known mutations causing genetic disorders are point mutations. The most common methods for detecting nucleotide changes involve the use of allele-specific oligonucleotides either as hybridization probes for PCR-amplified DNA templates (Saiki et al.,

Received May 19, 1997; Revised version accepted July 15, 1997.

* To whom correspondence should be addressed. 
1986) or as primers in PCR (Wu et al., 1989). Restriction analysis of PCRamplified products can also be used. However, the identification of undefined mutations in PCR-amplified DNA is still time-consuming. Single stranded conformation polymorphism (SSCP) is one of the most common methods used to screen mutations in a PCR amplified product (Orita et al., 1989). Several other methods have been used to detect nucleotide changes within an amplified product based on the observation of mobility shifts upon gel electrophoresis (Scholz et al., 1993; Sheffield et al., 1989; Yoshino et al., 1991). However, the aforementioned methods have two disadvantages: 1 , some nucleotide changes go undetected and 2, the nucleotide change is only roughly localized within the fragment (usually more than $100 \mathrm{bp}$ ) of the amplified product.

In order to establish a comprehensive scanning system for mutations in $\mathrm{X}$-linked recessive diseases, we developed multiplex $\underline{\mathrm{P} C R}$ using multiple tandem forward primers and a common reverse primer (abbreviated as MPTP from underlined letters). MPTP is based on the fact that a very short primer can recognize one nucleotide difference on the target sequence, and thus fails to hybridize. The tandem forward primers cover the entire target region and result in the amplification of a ladder of multiple fragments. A nucleotide change in the primer annealing region results in the disappearance of one or two amplified products. The significant advantage of our method is that all sequence variations in a target region of genes on X-chromosome can be localized to a narrow region after two PCR steps.

Glucose 6 phosphate dehydrogenase (G6PD) is an X-chromosome-encoded enzyme that catalyzes the first step in the pentose phosphate pathway. G6PD deficiency is common in malaria endemic regions and is estimated to affect upwards of 400 million people world-wide. Deficient subjects are mostly asymptomatic, but clinical manifestations range from neonatal jaundice due to acute hemolytic anemia to severe chronic nonspherocytic hemolytic anemia. To date, more than 300 different G6PD variants have been distinguished on the basis of biochemical parameters, suggesting a vast genetic heterogeneity (Beutler and Kuhl, 1990). So far, however, only a small portion of this heterogeneity has been confirmed at the DNA level through the identification of about 60 different point mutations in the G6PD coding sequence (Ganczakowski et al., 1995). To facilitate clarification of the molecular pathogenesis of G6PD deficiency, a fast and comprehensive scanning method to identity mutations is essential.

Here, we report that MPTP can be used to scan for mutations in exon 12 of the G6PD gene from G6PD deficiency patients. This simple method facilitates the molecular study of G6PD deficiency, especially in developing countries where it is common. 


\section{SUBJECTS AND METHODS}

Subjects. Blood samples from G6PD deficient patients were obtained from newborn male babies born at the Singapore National University Hospital. They were identified as having G6PD deficiency by neonatal screening (Saha et al, 1994). Blood samples were also obtained from a normal Japanese male as a control. Genomic DNA from these blood samples was extracted by the standard phenol chloroform method.

MPTP scanning. To evaluate MPTP, mutation scanning was performed in the region of the G6PD gene corresponding to exon 12, one of the mutation hot spots in Singaporean G6PD deficient patients (Saha et al., 1994). In MPTP, PCR is conducted twice. The first PCR is carried out to obtain sufficient target DNA and the second PCR is used for multiplex PCR.

The exon 12-encompassing region of the G6PD gene was first PCR amplified by using a set of primers hybridizing in introns 11 and 12 (Fig. 1a). The sequences of the primers and the PCR conditions were as described before (Hirono et al., 1994). The PCR product was then confirmed by electrophoresis using a $4 \%$ agarose

a

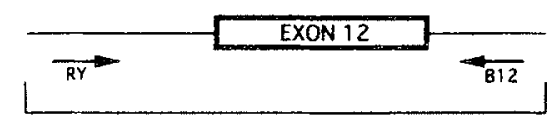

b

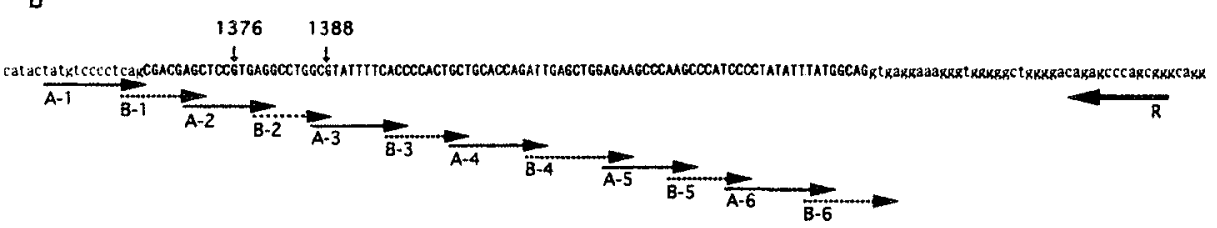

Fig. 1. Schematic presentation of PCR amplified region. a. PCR amplification of the region encompassing exon 12 of the G6PD gene. The exon 12-containing fragment was amplified from genomic DNA by using primers based on flanking introns. The box represents exon 12, and lines represent introns 11 and 12. The location and direction of primers are indicated by horizontal arrows. Primers RY and B12 were prepared as described previously (Hirono et al., 1994). The bracket indicates the amplified product ( $255 \mathrm{bp}$ ). b. MPTP, 12 different forward primers ranging from 13 to 15 mers (A-1 to 6 and B-1 to 6) and a common reverse primer $(R)$ were synthesized. Forward primers were designed to be in tandem and to cover the entire exon 12 region with 3 to $4 \mathrm{nt}$ overlaps. The location of primers is represented by arrows. G6PD Canton and Kaiping are shown by vertical arrows at nt 1376 and 1388 , respectively.

Vol. 42, No, 3, 1997 
gel (MetaPhor/Seaken HGT $3: 1$ ) containing $0.5 \mu \mathrm{g} / \mathrm{ml}$ ethidium bromide along with $2 \mathrm{mg}$ of a DNA size marker (50 bp ladder, Pharmacia LKB Biotechnology AB, Sweden).

In the second round PCR, each of the multiple forward primers was designed to work with one common reverse primer (Fig. lb). Primers for MPTP were designed so that the free energy of duplex formation $(\Delta G)$ was $-25 \pm 1 \mathrm{kcal} / \mathrm{mol}$ (Breslauer et al. 1986). To cover the entire region of exon 12 in the G6PD gene, 12 forward primers, each of $13-15$ bases were designed so that they were tandemly arranged and overlapped by 3-4 nucleotides (Fig. 1b). Since the amplified products differ by at most 10 nucleotides, it is difficult to separate them all by agarose gel electrophoresis. Therefore, six alternative primers (A-1 to 6 and B-1 to 6 in Fig. lb) were each mixed with one common reverse primer $(R)$ in two separate PCR reactions so that adjacent bands could be clearly separated by agarose gel electrophoresis.

Since G6PD Canton (GI376T) and Kaiping (GI388A) are common in Singapore (Saha et al., 1994), the applicability of MPTP to scan for known mutations was examined with another combination of primers comprising A-1, 3 and 5 as a multiplex forward primer and $\mathrm{R}$ primer as the common reverse primer (reaction C) (Fig. lb).

MPTP was carried out by the hot start PCR method. Ten microliters of solution containing $8 \mu \mathrm{l}$ of a muster mixture $(10 \mathrm{mM}$ Tris- $\mathrm{HCl} \mathrm{pH} 8.3,10 \mathrm{mM} \mathrm{KCl}$ $250 \mu \mathrm{M}$ dNTPs, $2.5 \mathrm{mM} \mathrm{MgCl}{ }_{2}$ and 5\% DMSO), $1 \mu 1$ of primer mixture, $1 \mu \mathrm{l}$ of the first PCR product and another $10 \mu 1$ of solution containing $8 \mu 1$ of the muster mixture, $1.9 \mu 1$ of the distilled water and $0.1 \mu 1$ of 5 U Ampli Taq DNA Polymerase Stoffel Fragment (Perkin-Elmer Co., U.S.A.) were pre-heated to $85^{\circ} \mathrm{C}$ individually and then mixed together at $85^{\circ} \mathrm{C}$. PCR was, then carried out with the following cycles: 25 cycles at $95^{\circ} \mathrm{C}$ and $72^{\circ} \mathrm{C}$ for $30 \mathrm{sec}$ each. The products were electrophoresed on $4 \%$ agarose gel (MetaPhor/Seaken HGT $3: 1$ ) containing $0.5 \mu \mathrm{g} / \mathrm{ml}$ ethidium bromide and the gels were photographed using a UV transilluminator.

Sequencing. The exon 12-encompassing region of the G6PD gene was amplified from all DNA samples and subjected to direct sequencing using a dye terminator kit (Perkin-Elmer Co.) and an automated DNA sequencer (ABI PRISM 310 Genetic Analyzer, Perkin-Elmer Co.).

\section{RESULTS AND DISCUSSION}

For a long time there has been a pressing need for a rapid method to scan for genomic mutations. We devised MPTP to facilitate such analyses, and applied it to scan for point mutations in exon 12 of the G6PD gene of G6PD-deficient patients. The full length of exon 12 of the G6PD gene was amplified as 12 different fragments in two separate amplification reaction (A and B). In each of the two reactions, 6 different size products were amplified from the control DNA that did 
not have a mutation in the amplified region (Fig. 2a lane C, Fig. 2b lane C).

Twelve DNA samples obtained from Singaporean patients with G6PD deficiency were examined for nucleotide changes by MPTP. In 5 out of 12 cases (cases $2,3,4,5$ and 11 ) we were unable to amplify a product corresponding to $126 \mathrm{bp}$ fragment in reaction A (Fig. 2a lanes 2, 3, 4, 5 and 11), suggesting the presence of a mutation within the forward primer recognition region between $\mathrm{nt} 1370$ and $\mathrm{nt}$ 1381. In accordance with this, DNA sequencing of the amplified fragments disclosed a $G$ to $T$ nucleotide change at $n t 1376$ (G1376T) in all of the five DNA samples. The sequence of all other regions scanned by MPTP was completely normal. The G1376T mutation, known as G6PD Canton, is the major mutation identified in G6PD deficient patients in Singapore (Saha et al., 1994).

The $110 \mathrm{bp}$ band of reaction A was missing in case number 7 (Fig. 2a lane 7), indicating a mutation between nt 1386 and 1398. In reaction $B$, the same case 7 lacked an amplified fragment of $117 \mathrm{bp}$, implying that a mutation may be present between nt 1379 and 1388. Combining these two results, case 7 was predicted to have a mutation in between $\mathrm{nt} 1386$ and 1388, where the two primers overlap. Sequencing analysis showed that a $G$ to $T$ nucleotide change was present at nt 1388 (G1388A). All other cases had six amplified products in reaction B and were found to have a wild-type exon 12 sequence.

These results showed that MPTP can be used to scan all mutations irrespective of their location in the primer annealing site. This method, which only requires a PCR amplification machine, is simple and enables many DNA samples to be handled at the same time. If a mutation is present, its location can be determined to within 13-15 nucleotides within a day, the period required to perform 2 PCR steps. Therefore, DNA sequencing, where appropriate, can be focused on a small region.

To facilitate the identification of the most common mutation causing G6PD deficiency in Singapore, we set up another PCR reaction involving three forward primers and a common reverse primer (reaction $C$ ). As expected, three products were obtained from the control DNA (Fig. 2c lane C). Cases 2 and 7 lacked the amplified products corresponding to $126 \mathrm{bp}$ and $110 \mathrm{bp}$, respectively. This indicated that by changing the combination of primers many kinds of mutations can be scanned in one reaction. Therefore, this scanning system with selected primers will facilitate mutation detection in localities where the spectrum of mutations of the G6PD gene is known.

Because MPTP does not need sophisticated machines, the method can be set up in almost any laboratory and should be useful in developing countries, where G6PD deficiency is especially common. Since MPTP accelerates analysis of G6PD deficiency at the DNA level, we anticipate that the full spectrum of mutations affecting the coding region of the G6PD gene in G6PD-deficient patients will soon be elucidated. 

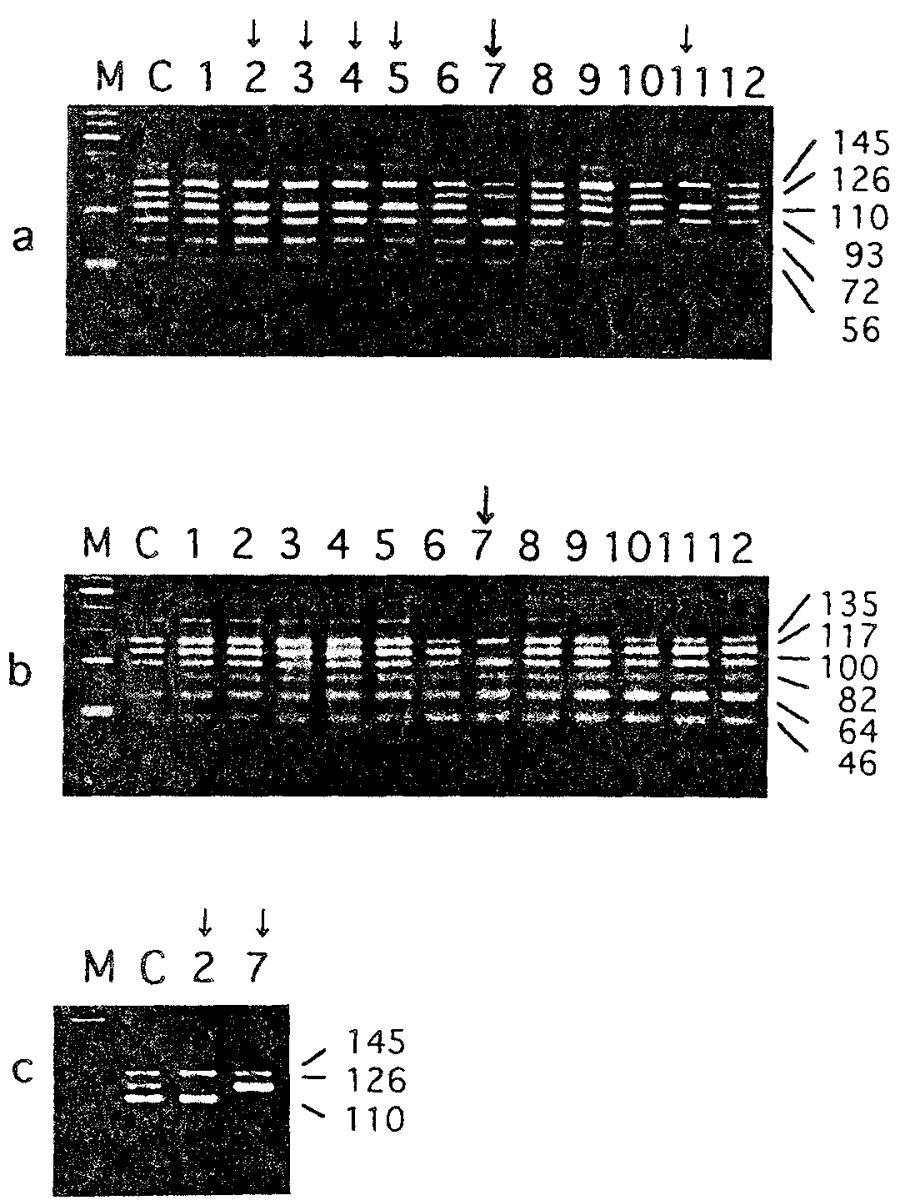

Fig. 2. Results of MPTP. a. MPTP amplified products of reaction A. b. MPTP amplified products of reaction B. c. MPTP amplified products of reaction C. Six forward primers (A-1 to 6 or B-1 to 6$)$ were designed to work together with one common reverse primer in MPTP (reactions $A$ and $B$ ) (Fig. 1). In reaction $A$, primers A-1, A-2, A-3, A-4, A-5, A-6 and $R$ were mixed at $0.1,0.3,0.4,0.4$, $0.4,0.4$ and $1.0 \mu$ mol/liter, respectively. In reaction $B$, primers $B-1, B-2, B-3$, B-4, B-5, B-6 and R were mixed at $0.1,1.0,0.8,0.2,0.8,0.6$ and $1.0 \mu \mathrm{mol} /$ liter, respectively. Reaction $\mathrm{C}$ has $0.1 \mu \mathrm{mol} /$ liter of $\mathrm{A}-1,0.5 \mu \mathrm{mol} / \mathrm{liter}$ of $\mathrm{A}-3,0.3$ $\mu \mathrm{mol} /$ liter of A-5 and $0.5 \mu \mathrm{mol} /$ liter of $\mathrm{R}$. Each of amplified products from G6PD cases (cases 1 to 12) was electrophoresed in the corresponding lanes in panels $a$ and $b$. The length of each PCR amplified product is indicated on the right side of the photographs. Six different fragments were amplified in reactions $\mathrm{A}$ and $\mathrm{B}$ from the control DNA (lanes $\mathrm{C}$ in panels $\mathrm{a}$ and $\mathrm{b}$ ). The $126 \mathrm{bp}$ amplified product disappeared from lanes 2, 3,5 and 11 of reaction $A$. The 100 and $117 \mathrm{bp}$ bands in reactions $A$ and $B$, respectively, disappeared from lanes 7 of panels $a$ and $b$. In reaction $C$, two DNA samples of cases 2 and 7 were examined for the same mutation. The 126 and 110 bp products disappeared, respectively (panel c). $M$ indicates a 100 bp DNA ladder. 
Acknowledgments We thank Dr. A. Pugsley for advice and for critically reading the manuscript, and Miss A. Maruyama for her help in preparing the manuscript. This work was supported by grants from the Japan Society for Promotion of Science, and the Ministry of Education, Science, Sports and Culture of Japan.

\section{REFERENCES}

Beutler E, Kuhl W (1990): The NT 1311 polymorphism of G6PD: G6PD Mediterranean mutation may have originated independently in Europe and Asia. Am J Hum Genet 47: 1008-1012

Breslauer KJ, Frank R, Blocker H, Marky KA (1986): Predicting DNA duplex stability from the base sequence. Proc Natl Acad Sci USA 83: 3746-3750

Ganczakowski M, Town M, Bowden DK, Vulliamy TJ, Kaneko A, Clegg JB, Weatherall DJ, Luzzatto L (1995): Multiple glucose 6-phosphate dehydrogenase-deficient variants correlate with malaria endemicity in the Vanuatu Archipelago (Southwestern pacific). Am J Hum Genet 56: 294-301

Hirono A, Miwa S, Fujii H, Ishida F, Yamada K, Kubota K (1994): Molecular study of eight Japanese cases of glucose-6-phosphate dehydrogenase deficiency by nonradioisotopic singlestrand conformation polymorphism analysis. Blood 83: 3363-3368

Orita M, Iwahana H, Kanazawa H, Hayashi K, Sekiya T (1989): Detection of polymorphisms of human DNA by gel electrophoresis as single-strand conformation polymorphisms. Proc Natl Acad Sci USA 86: $2766-2770$

Saha S, Saha N, Tay JSH, Jeyaseelan K, Basair JB, Chew SE (1994): Molecular characterisation of red cell glucose-6-phosphate dehydrogenase deficiency in Singapore Chinese. Am J Hematol 42: 273-277

Saiki RK, Bugawan TL, Horn GT, Mullis KB, Erlich HA (1986): Analysis of enzymatically amplified $\beta$-globin and HLA-DQa DNA with allele-specific oligonucleotide probes. Nature 324: $163-165$

Scholz RB, Milde-Langosch K, Jung R, Schlechte H, Kabisch H, Wagener C, Loning T (1993): Rapid screening for Tp53 mutations by temperature gradient gel electrophoresis: a comparison with SSCP analysis. Hum Mol Genet 2: 2155-2158

Sheffield V, Cox D, Lerman L, Myers R (1989): Attachment of a 40-base-pair G+C-rich sequence (GC-clamp) to genomic DNA fragments by the polymerase chain reaction results in improved detection of single-base changes. Proc Natl Acad Sci USA 86: 232-236

Wu DY, Ugozzoli L, Pal BK, Wallace RB (1989): Allele-specific enzymatic amplification of $\beta$-globin genomic DNA for diagnosis of sickle cell anemia. Proc Natl Acad Sci USA 86: 2757-2760

Yoshino K, Nishigaki K, Husimi Y (1991): Temperature sweep gel electrophoresis: a simple method to detect point mutations. Nucleic Acids Res 19: 3153 